\title{
THE ULTRAVIOLET-TO-MID-INFRARED SPECTRAL ENERGY DISTRIBUTION OF WEAK EMISSION LINE QUASARS
}

\author{
Ryan A. Lane ${ }^{1}$, Ohad Shemmer ${ }^{1}$, Aleksandar M. Diamond-Stanic ${ }^{2,10}$, Xiaohui Fan ${ }^{3}$, Scott F. Anderson ${ }^{4}$, \\ W. N. Brandt ${ }^{5,6}$, Richard M. Plotkin ${ }^{7}$, Gordon T. Richards ${ }^{8}$, Donald P. Schneider ${ }^{5}$, and Michael A. Strauss ${ }^{9}$ \\ ${ }^{1}$ Department of Physics, University of North Texas, Denton, TX 76203, USA; RyanLane@ my.unt.edu, ohad@unt.edu \\ ${ }^{2}$ Center for Astrophysics and Space Sciences, University of California, San Diego, La Jolla, CA 92093, USA \\ ${ }^{3}$ Steward Observatory, University of Arizona, 933 North Cherry Avenue, Tucson, AZ 85721, USA \\ ${ }^{4}$ Department of Astronomy, University of Washington, Box 351580, Seattle, WA 98195, USA \\ ${ }^{5}$ Department of Astronomy \& Astrophysics, The Pennsylvania State University, University Park, PA 16802, USA \\ ${ }^{6}$ Institute for Gravitation and the Cosmos, The Pennsylvania State University, University Park, PA 16802, USA \\ 7 Astronomical Institute “Anton Pannekoek," University of Amsterdam, Science Park 904, NL-1098 XH Amsterdam, The Netherlands \\ ${ }^{8}$ Department of Physics, Drexel University, 3141 Chestnut Street, Philadelphia, PA 19104, USA \\ ${ }^{9}$ Princeton University Observatory, Peyton Hall, Princeton, NJ 08544, USA \\ Received 2011 June 2; accepted 2011 September 23; published 2011 December 2
}

\begin{abstract}
We present Spitzer Space Telescope photometry of 18 Sloan Digital Sky Survey (SDSS) quasars at $2.7 \leqslant z \leqslant 5.9$ which have weak or undetectable high-ionization emission lines in their rest-frame ultraviolet (UV) spectra (hereafter weak-lined quasars, or WLQs). The Spitzer data are combined with SDSS spectra and ground-based, near-infrared (IR) photometry of these sources to produce a large inventory of spectral energy distributions (SEDs) of WLQs across the rest-frame $\sim 0.1-5 \mu \mathrm{m}$ spectral band. The SEDs of our sources are inconsistent with those of BL Lacertae objects which are dominated by synchrotron emission due to a jet aligned close to our line of sight, but are consistent with the SED of ordinary quasars with similar luminosities and redshifts that exhibit a near-to-mid-IR "bump," characteristic of hot dust emission. This indicates that broad emission lines in WLQs are intrinsically weak, rather than suffering continuum dilution from a jet, and that such sources cannot be selected efficiently from traditional photometric surveys.
\end{abstract}

Key words: galaxies: active - galaxies: nuclei - infrared: galaxies - quasars: emission lines - quasars: general

\section{INTRODUCTION}

The spectra of most optically selected quasars are marked by strong, broad emission lines in the rest-frame ultraviolet (UV) band. However, the Sloan Digital Sky Survey (SDSS; York et al. 2000) has, so far, discovered $\sim 80$ quasars at $z \gtrsim 2.2$ with weak or undetectable rest-frame UV emission lines (hereafter weak-lined quasars, or WLQs), starting with the prototype SDSS J153259.96-003944.1 (Fan et al. 1999; see also Anderson et al. 2001; Collinge et al. 2005; Diamond-Stanic et al. 2009, hereafter DS09; Plotkin et al. 2010a). Such WLQs can be defined as sources having rest-frame equivalent widths (EWs) of $\leqslant 15.4 \AA$ for the $\mathrm{Ly} \alpha+\mathrm{N} v$ emission complex; this threshold represents the $3 \sigma$ limit at the low-EW end of the lognormal distribution of EW[Ly $\alpha+\mathrm{Nv}]$ in $z>3$ SDSS quasars (DS09). Due to their largely featureless spectra, the redshifts of these sources can only be determined reliably from the onset of the Ly $\alpha$ forest (i.e., $\lambda_{\text {rest }} \sim 1200 \AA$ ). The discovery of WLQs only at $z \gtrsim 2.2$ is clearly a selection effect, as the onset of the Ly $\alpha$ forest emerges in the SDSS spectral range at that redshift. Indeed, there are indications for a population of lower-redshift sources with similar characteristics to the high-redshift WLQ population (e.g., McDowell et al. 1995; Leighly et al. 2007b; Hryniewicz et al. 2010, Plotkin et al. 2010b).

Since their discovery, a number of scenarios have been proposed to explain the properties of these remarkable objects, although many have been subsequently shown to be unlikely. For example, WLQs are unlikely to be broad absorption line (BAL) quasars, as their spectra do not show obvious broad C IV absorption features. In addition, Shemmer et al. (2006,

\footnotetext{
${ }^{10}$ Center for Galaxy Evolution Fellow.
}

hereafter S06) show that WLQs are not likely to be active galactic nuclei (AGNs) where emission lines have been obscured by dust and that they are not normal galaxies with luminosities that have been amplified by gravitational lensing. Spectroscopic monitoring of four WLQs further suggests that it is unlikely that the weakness of their lines can be explained by microlensing that temporarily and preferentially amplifies the continuum relative to the broad emission lines (DS09). Instead, S06 proposed that WLQs are either the long-sought, high-redshift counterparts to BL Lacertae (BL Lac) objects (e.g., Stocke \& Perrenod 1981) or that they are "ordinary" unbeamed, unobscured quasars with extreme properties such as a deficit of line-emitting gas in the broad emission line region (BELR) or an extremely high accretion rate (see also Fan et al. 1999). It has also been speculated that WLQs may represent an early or transitional phase in quasar evolution where quasar activity just recently "switched on" (Hryniewicz et al. 2010; see also Liu \& Zhang 2011).

Classical BL Lac objects are a rare class of AGNs with nearly featureless spectra that are radio loud, X-ray strong, polarized, and highly variable. All these characteristics are attributed to the fact that BL Lac objects are AGNs viewed along their powerful, narrow jets (e.g., Urry \& Padovani 1995). In particular, the nearly featureless spectrum of BL Lac objects is a consequence of relativistically boosted synchrotron emission from the jet that overwhelms the characteristic AGN emission lines. In contrast, WLQs are, at most, radio and X-ray intermediate, exhibiting only minimal overlap with the radio- and X-ray-weak tails of the BL Lac population (e.g., Shemmer et al. 2009, hereafter S09). Furthermore, unlike BL Lac objects, WLQs do not display significant polarization or variability (DS09). These properties alone are a strong, but not conclusive, argument against the 
Table 1

Basic Properties of the New WLQ Sample

\begin{tabular}{|c|c|c|c|c|c|}
\hline $\begin{array}{l}\text { Quasar } \\
\text { (SDSS J) }\end{array}$ & $z$ & $A B_{1445(1+z) \AA}$ & $\begin{array}{l}\log v L_{v}{ }^{\mathrm{a}} \\
\left(\operatorname{erg} \mathrm{s}^{-1}\right)\end{array}$ & $R^{\mathrm{b}}$ & Reference \\
\hline $004054.65-091526.8^{d}$ & 5.0 & 18.79 & 47.0 & $<3$ & 1 \\
\hline $031712.23-075850.4$ & 2.7 & 18.68 & 46.6 & $<12^{\mathrm{c}}$ & 1 \\
\hline $085332.78+393148.8$ & 4.2 & 20.24 & 46.3 & 35 & 2 \\
\hline $114153.34+021924.3^{\mathrm{d}}$ & 3.5 & 18.50 & 46.9 & 12 & 1 \\
\hline $120715.46+595342.9$ & 4.5 & 19.95 & 46.5 & $<8$ & 2 \\
\hline $121221.56+534128.0^{\mathrm{d}}$ & 3.1 & 18.63 & 46.7 & $<2$ & 1 \\
\hline $123743.08+630144.9^{\mathrm{d}}$ & 3.4 & 18.97 & 46.7 & $<3$ & 1 \\
\hline $130332.42+621900.3^{\mathrm{d}}$ & 4.6 & 20.10 & 46.4 & $<10$ & 2 \\
\hline $133219.66+622716.0$ & 3.2 & 19.17 & 46.5 & 33 & 1 \\
\hline $133422.63+475033.6^{d}$ & 5.0 & 18.92 & 46.9 & $<4$ & 2 \\
\hline $133550.81+353315.8$ & 5.9 & 19.98 & 46.6 & $<9$ & 3 \\
\hline $135249.82-031354.3$ & 4.7 & 19.40 & 46.7 & $<5$ & 2 \\
\hline $140300.22+432805.4^{\mathrm{d}}$ & 4.7 & 19.32 & 46.7 & $<5$ & 2 \\
\hline $154734.95+444652.5$ & 4.5 & 19.82 & 46.5 & $<8$ & 2 \\
\hline
\end{tabular}

Notes.

a Monochromatic luminosity averaged over a $40 \AA$ wide bin centered on a rest-frame wavelength of $1445 \AA$, corrected for Galactic extinction.

b Radio loudness parameter; see Section 2.1. Flux densities at a rest-frame wavelength of $4400 \AA$ were obtained by extrapolation of the flux densities at a rest-frame wavelength of $1445 \AA$ assuming an optical continuum of the form $f_{v} \propto v^{-0.5}$. Unless otherwise noted, flux densities at a rest-frame frequency of $5 \mathrm{GHz}$ were computed from the flux densities at an observed-frame wavelength of $20 \mathrm{~cm}$, obtained from the Faint Images of the Radio Sky at Twenty-Centimeters (FIRST) survey (Becker et al. 1995), using a radio continuum of the form $f_{v} \propto v^{-0.5}$; upper limits on $R$ were calculated according to the $3 \sigma$ FIRST detection threshold at the source position.

${ }^{c}$ Upper limit was calculated according to the NRAO/VLA Sky Survey (Condon et al. 1998) detection threshold of $2.5 \mathrm{mJy}$.

d Source formally classified as a WLQ at $3.00 \leqslant z \leqslant 5.41$ by DS09 and is listed in their Table 2 .

References. (1) Collinge et al. 2005; (2) Schneider et al. 2005; (3) Fan et al. 2006.

BL Lac hypothesis for explaining the weak lines in WLQs, given that radio-quiet BL Lac objects must be extraordinarily rare, if they exist at all (Stocke et al. 1990, and see also Jannuzi et al. 1993; Londish et al. 2004; Plotkin et al. 2010b).

The "BL Lac" and "extreme quasar" scenarios provide differing predictions for the shape of the UV-to-mid-infrared (IR) spectral energy distributions (SEDs) of WLQs. The BL Lac emission in this range is mostly due to synchrotron emission; the synchrotron radiation peaks either in the UV/soft-X-ray bands for high-energy peaked BL Lac objects (HBLs), or in the nearIR band for low-energy peaked BL Lac objects (LBLs; e.g., Padovani \& Giommi 1995; Fossati et al. 1998; Nieppola et al. 2006). The prominent LBL near-IR peak dominates the UVto-mid-IR SED, while an HBL-like SED in this spectral range is characterized by a power law representing the tail of the synchrotron peak. In contrast, ordinary quasars' SEDs display the characteristic "dip" at $\sim 1 \mu \mathrm{m}$ between the emissions from the disk and the circumnuclear heated dust (e.g., Elvis et al. 1994). DS09 traced the UV-to-mid-IR SEDs of four WLQs (SDSS J1302+0030 at $z=4.5$, SDSS J1408+0205 at $z=4.0$, SDSS J1442+0110 at $z=4.5$, and SDSS J1532-0039 at $z=4.6)$ using Spitzer Space Telescope mid-IR photometry as well as archival SDSS spectra and ground-based, near-IR photometry. All their SEDs deviate significantly from a pure power-law, HBL-like continuum, and none can be fitted with an LBL-like spectrum. Instead, their results indicate the presence of hot ( $T \sim 1000 \mathrm{~K})$ dust emission in all four sources, consistent with the SEDs of ordinary quasars.

In this work, we extend the near-to-mid-IR photometry to a statistically representative sample of 18 WLQs, including the four DS09 sources, and present conclusive evidence against the possibility that a relativistically boosted continuum dilutes the WLQ line emission. We describe our observations in Section 2 and present our basic findings, including a composite UV-to-mid-IR SED of WLQs, in Section 3. We discuss our results in Section 4, and present our conclusions in Section 5. Throughout the text, shortened versions of object names are used and full names appear in the tables and figures. Luminosity distances are computed using the standard cosmological model $\left(\Omega_{\Lambda}=0.7, \Omega_{\mathrm{M}}=0.3\right.$, and $H_{0}=70 \mathrm{~km} \mathrm{~s}^{-1} \mathrm{Mpc}^{-1}$ ).

\section{OBSERVATIONS AND DATA REDUCTION}

\subsection{New Spitzer Observations}

We have selected a representative sample of 14 high-redshift $(2.7 \leqslant z \leqslant 5.9)$ WLQs that were bright enough for economical Spitzer Space Telescope (Werner et al. 2004) photometry. ${ }^{11}$ Except for SDSS J1335+3533, for which the rest-frame UV spectrum was obtained from Fan et al. (2006), our sources' spectra were obtained from the SDSS Data Release 3 quasar catalog (Schneider et al. 2005). These sources represent the ranges in luminosity and redshift as well as the range in radio loudness observed in the WLQ population (none of our sources are radio loud, i.e., all have $R<100$, where $R=f_{5 \mathrm{GHz}} / f_{4400 \AA}$; Kellermann et al. 1989). In fact, our sample constitutes the majority of the WLQ population that were known at the time; it also remains representative of the larger WLQ population known today (e.g., DS09; Plotkin et al. 2010a). Basic properties of the sources are given in Table 1, and their observed-frame UV spectra are displayed in Figure 1.

We conducted Spitzer observations of the 14 WLQs using the InfraRed Array Camera (IRAC; Fazio et al. 2004) and the

\footnotetext{
11 Source selection was based on our early SDSS measurements; only seven of these sources are formally classified as WLQs by the DS09 study of SDSS quasars at $3.00 \leqslant z \leqslant 5.41$; see Table 1 .
} 

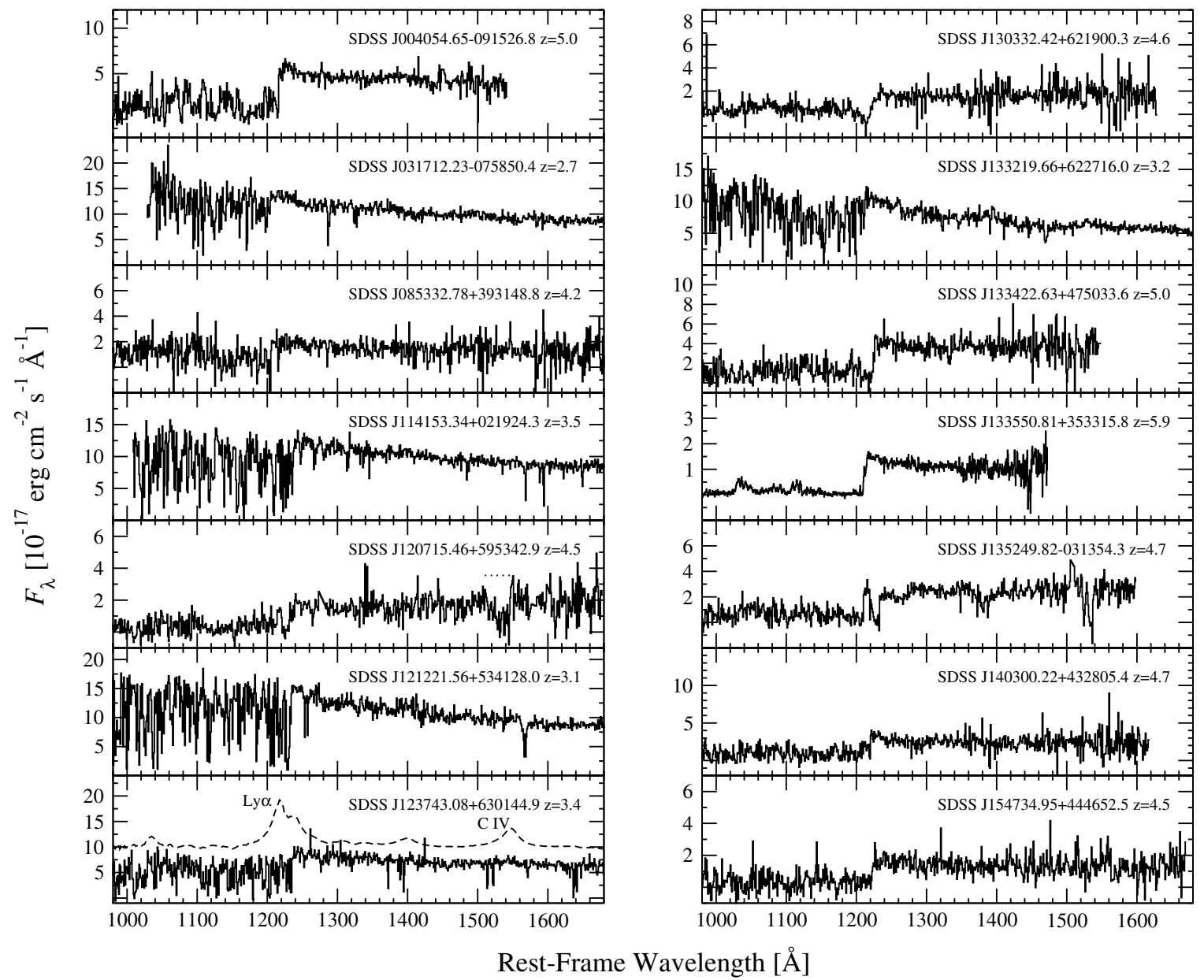

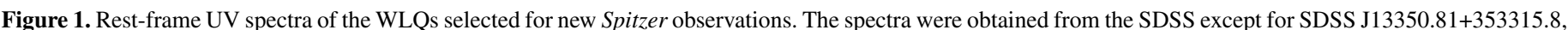

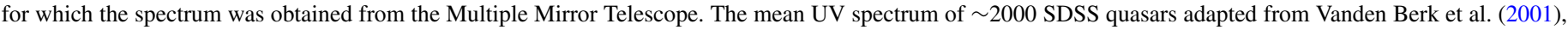

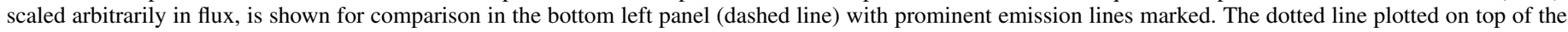
spectrum of SDSS J120715.46+595342.9 marks the C IV BAL trough.

Multiband Imaging Photometer for Spitzer (MIPS; Rieke et al. 2004) in Cycle 3 (Program ID 30476). Each WLQ was observed with IRAC in the $3.6 \mu \mathrm{m}, 4.5 \mu \mathrm{m}, 5.8 \mu \mathrm{m}$, and $8.0 \mu \mathrm{m}$ bands and with MIPS in the $24 \mu \mathrm{m}$ band. Total integration times for the IRAC exposures range between $\sim 400$ and $\sim 2000 \mathrm{~s}$ with $100 \mathrm{~s}$ per frame, per source; the total integration times for the MIPS exposures range between $\sim 400$ and $\sim 2100$ s with 30 s per frame, per source. These exposures were designed to achieve a signalto-noise ratio of $\sim 20$ for each channel. The Spitzer observation log is presented in Table 2.

Our analysis of the IRAC and MIPS observations is based on the Basic Calibrated Data (BCD) images from version S18.5.0 of the Spitzer Science Center (SSC) pipeline. The Spitzer-specific software package MOPEX (version 18.2.2; Makovoz \& Marleau 2005) was used to generate mosaics from the BCD images using background matching and outlier pixel rejection. These mosaics excluded the first image for each IRAC channel and the first three images for MIPS, for each target, due to the "firstframe effect" (see, e.g., the IRAC Instrument Handbook ${ }^{12}$ and the MIPS Instrument Handbook ${ }^{13}$ ). Aperture photometry was performed on the new mosaics using APEX, a Spitzer-specific photometry routine incorporated into MOPEX, using, for all IRAC channels, a source aperture radius of $3 . \prime 6$ (3 pixels) with the

\footnotetext{
12 http://ssc.spitzer.caltech.edu/irac/iracinstrumenthandbook/home

$13 \mathrm{http}: / / \mathrm{ssc}$.spitzer.caltech.edu/mips/mipsinstrumenthandbook/home
}

background measured in a source-free annulus (centered on the source) with an inner radius of $14^{\prime \prime} .4$ and a width of 7.2 ; and using, for the MIPS $24 \mu \mathrm{m}$ channel, a source aperture radius of 6.' 1 (2.5 pixels) with the background measured in a source-free annulus (centered on the source) with an inner radius of $20^{\prime \prime}$ and a width of $12^{\prime \prime} .2$.

The IRAC fluxes were corrected for source aperture size using the aperture correction factors from the IRAC Instrument Handbook. Array-location-dependent corrections were applied to the IRAC data but no color or pixel-phase corrections were applied as these were found to have an effect of $<1 \%$ on the total flux. For the MIPS data, aperture corrections were calculated using the MIPS $24 \mu \mathrm{m}$ point-spread function (PSF), and the fluxes were further increased by $4 \%$ to correct for an $f_{v} \propto v^{-1}$ power law which represents an ordinary quasar SED in the $\sim 4-6 \mu \mathrm{m}$ rest-frame band (corresponding to $24 \mu \mathrm{m}$ in the observed frame for the redshifts of our sources; e.g., Elvis et al. 1994; Richards et al. 2006, hereafter R06). We note, however, that our main results, presented in Section 3, would not have changed significantly had this small correction not been applied. Table 3 presents the results of our Spitzer photometry. The statistical flux uncertainties for all mosaics are smaller than the 5\% calibration accuracy of IRAC and the $4 \%$ calibration accuracy of MIPS; however, we quote a conservative $5 \%$ uncertainty for all our flux density values in Table 3. 
Table 2

Spitzer Observation Log

\begin{tabular}{|c|c|c|c|c|c|c|c|c|c|}
\hline \multirow{2}{*}{$\begin{array}{l}\text { Quasar } \\
\text { (SDSS J) }\end{array}$} & \multirow[b]{2}{*}{ IRAC Campaign } & \multirow[b]{2}{*}{ MJD } & \multicolumn{4}{|c|}{ IRAC Integration Time (s) } & \multirow[b]{2}{*}{ MIPS Campaign } & \multirow[b]{2}{*}{ MJD } & \multirow{2}{*}{$\begin{array}{c}\text { MIPS } 24 \mu \mathrm{m} \\
\text { Integration Time (s) }\end{array}$} \\
\hline & & & $3.6 \mu \mathrm{m}$ & $4.5 \mu \mathrm{m}$ & $5.8 \mu \mathrm{m}$ & $8.0 \mu \mathrm{m}$ & & & \\
\hline $004054.65-091526.8$ & IRAC008900 & 53960.02 & 774 & 871 & 774 & 796 & MIPS007600 & 53939.36 & 742 \\
\hline $031712.23-075850.4$ & IRAC009500 & 54147.18 & 484 & 581 & 484 & 515 & MIPS012600 & 54339.14 & 402 \\
\hline $085332.78+393148.8$ & IRAC009700 & 54227.04 & 1452 & 1531 & 1452 & 1404 & MIPS010700 & 54076.87 & 1980 \\
\hline $114153.34+021924.3$ & IRAC009800 & 54284.85 & 387 & 484 & 387 & 421 & MIPS012000 & 54292.42 & 371 \\
\hline $120715.46+595342.9$ & IRAC009200 & 54069.68 & 1452 & 1549 & 1403 & 1451 & MIPS010700 & 54077.76 & 2103 \\
\hline $121221.56+534128.0$ & IRAC009300 & 54096.08 & 387 & 484 & 387 & 421 & MIPS010700 & 54077.71 & 402 \\
\hline $123743.08+630144.9$ & IRAC009200 & 54066.94 & 484 & 580 & 484 & 515 & MIPS010700 & 54077.70 & 402 \\
\hline $130332.42+621900.3$ & IRAC009200 & 54069.65 & 1161 & 1259 & 1161 & 1170 & MIPS010700 & 54077.68 & 1701 \\
\hline $133219.66+622716.0$ & IRAC009300 & 54095.59 & 580 & 678 & 581 & 608 & MIPS010700 & 54077.67 & 804 \\
\hline $133422.63+475033.6$ & IRAC009500 & 54150.81 & 968 & 1065 & 968 & 983 & MIPS011900 & 54249.74 & 1238 \\
\hline $133550.81+353315.8$ & IRAC009500 & 54151.25 & 1937 & 2032 & 1936 & 1918 & MIPS012000 & 54305.04 & 2567 \\
\hline $135249.82-031354.3$ & IRAC008900 & 53961.30 & 968 & 1065 & 968 & 983 & MIPS007600 & 53938.85 & 1237 \\
\hline $140300.22+432805.4$ & IRAC009500 & 54150.23 & 1161 & 1259 & 1161 & 1170 & MIPS011900 & 54250.75 & 1640 \\
\hline $154734.95+444652.5$ & IRAC008900 & 53961.23 & 1161 & 1258 & 1161 & 1170 & MIPS011800 & 54203.29 & 1670 \\
\hline
\end{tabular}

\subsection{Archival Spitzer Data}

We also include Spitzer photometry of the four WLQs from DS09 in Table 3. We have reanalyzed the Spitzer data for three of these sources using the processes described in Section 2.1. A nearby star prevents accurate aperture photometry on SDSS J1532-0039 (see DS09); thus we performed PSF fitting for this source employing standard IRAF ${ }^{14}$ photometry tasks on the mosaics generated by MOPEX. The only significant difference we find with the DS09 fluxes for their four WLQs is for the MIPS $24 \mu \mathrm{m}$ flux of the faintest source, SDSS J1442+0110, which we find to be higher by a factor of 1.7 in our new analysis. We attribute this difference to an improved mosaicking technique, which uses an updated version of the SSC pipeline; this discrepancy does not affect the main conclusions of either study.

\subsection{New Near-IR Observations}

Near-IR observations were taken with the Astrophysical Research Consortium $3.5 \mathrm{~m}$ telescope at the Apache Point Observatory for two of our sources, SDSS J1212+5341 (2008 June 15 and 2009 February 14) and SDSS J1237+6301 (2009 February 14). Images were taken in the $J, H$, and $K_{\mathrm{S}}$ filters with the Near-Infrared Camera \& Fabry-Perot Spectrometer. In the $J$ filter, $15 \times 60 \mathrm{~s}$ sub-exposures were taken over a five-point dither pattern, for a total exposure time of $900 \mathrm{~s}$ for each source on each night. For the $H$ and $K_{\mathrm{S}}$ filters, 12 s sub-exposures were used over two sets of dither patterns with 25 sub-exposures each for a total exposure time of $600 \mathrm{~s}$ per filter on each night. Each sub-exposure was Fowler-sampled eight times to reduce the read noise. Sky maps were created for each dither pattern by taking the median of the sub-exposures. Both $12 \mathrm{~s}$ and $60 \mathrm{~s}$ dark images were taken at the beginning of each night. To create the flat field, we subtracted the dark images from the sky maps and then normalized them so that the flat field had an average value of 1 count. After sky subtraction and flat fielding, the images were aligned (using 3-4 reference stars) and then coadded with standard tasks in IRAF. Differential photometry was then performed using the Two Micron All Sky Survey (Skrutskie et al. 2006) photometry of four field stars in the $4.6 \times 4.6$ field

\footnotetext{
14 Image Reduction and Analysis Facility (IRAF) is distributed by the National Optical Astronomy Observatory, which is operated by AURA, Inc., under cooperative agreement with the National Science Foundation.
}

of view of each source. Each field star was chosen to be near the target WLQ and of similar brightness. Finally, we corrected the photometric data for Galactic extinction by applying the standard extinction curve from Cardelli et al. (1989), using the extinction maps of Schlegel et al. (1998). The $J, H$, and $K_{\mathrm{S}}$ photometric data for these two WLQs are presented in Table 4.

\section{SPECTRAL ENERGY DISTRIBUTION OF WLQs}

\subsection{UV-to-mid-IR SEDs of Individual WLQs}

In Figure 2, we present the UV-to-mid-IR SEDs of the 18 WLQs observed by Spitzer. The SEDs are composed of the Spitzer photometry from Table 3 as well as near-IR photometry for six of the sources, two sources from Table 4 and four sources from DS09 ${ }^{15}$ (see Section 1). The rest-frame UV spectral band in the SEDs was obtained from the WLQs' SDSS spectra, with the exceptions of SDSS J1532-0039 and SDSS $\mathrm{J} 1335+3533$, for which the UV bands were obtained from spectra taken with the Low-Resolution Imaging Spectrometer at the W. M. Keck Observatory (Oke et al. 1995) and with the Red Channel Spectrograph ${ }^{16}$ on the Multiple Mirror Telescope, respectively. The rest-frame UV spectra were corrected for Galactic extinction using the same procedures as in Section 2.3. The flux densities were averaged over line-free, $40 \AA$ wide bands centered at rest-frame wavelengths $1290 \AA, 1350 \AA, 1445 \AA$, $1695 \AA, 1830 \AA$, and $1965 \AA$, depending on source redshift (see Table 5). Table 5 also provides the power-law indices $\left(\alpha_{\nu}\right)$ calculated from fitting a power law of the form $f_{v} \propto v^{-\alpha_{v}}$ to the average fluxes in the rest-frame UV photometric bins. Our $\alpha_{v}$ values lie in the range $0.28<\alpha_{v}<2.90$ with a mean and standard deviation of 1.12 and 0.73 , respectively. These WLQs therefore have rest-frame UV continua that are consistent, on average, with the respective continua of ordinary SDSS quasars at $3.6<z<5.0$ (comparable to the redshift range of our WLQs) that show power-law indices of $\alpha_{v}=0.79 \pm 0.34$ (Fan et al. 2001).

Inspection of Figure 2 clearly indicates that a power-law model ${ }^{17}$ cannot represent the entire UV-to-mid-IR SED of any of

\footnotetext{
15 The near-IR photometry for the four DS09 sources was corrected for Galactic extinction following the procedures outlined in Section 2.3.

16 http://www.mmto.org/instruments/rcupdate.shtml

17 Where the power-law index, $\alpha_{\lambda}$, is defined as $\lambda f_{\lambda} \propto \lambda^{-\alpha_{\lambda}}$; see Table 6 .
} 
Table 3

Spitzer Photometry for the Entire WLQ Sample

\begin{tabular}{|c|c|c|c|c|c|}
\hline \multirow{2}{*}{$\begin{array}{l}\text { Quasar } \\
\text { (SDSS J) }\end{array}$} & \multicolumn{5}{|c|}{ Flux Density $(\mu \mathrm{Jy})$} \\
\hline & $3.6 \mu \mathrm{m}$ & $4.5 \mu \mathrm{m}$ & $5.8 \mu \mathrm{m}$ & $8.0 \mu \mathrm{m}$ & $24.0 \mu \mathrm{m}$ \\
\hline $004054.65-091526.8$ & $101.0 \pm 5.1$ & $79.6 \pm 4.0$ & $75.0 \pm 3.8$ & $110.0 \pm 5.5$ & $509 \pm 26$ \\
\hline $031712.23-075850.4$ & $135.0 \pm 6.8$ & $141.0 \pm 7.1$ & $186.0 \pm 9.3$ & $335 \pm 17$ & $610 \pm 31$ \\
\hline $085332.78+393148.8$ & $38.0 \pm 1.9$ & $29.0 \pm 1.5$ & $36.6 \pm 1.8$ & $53.5 \pm 2.7$ & $605 \pm 30$ \\
\hline $114153.34+021924.3$ & $236 \pm 12$ & $235 \pm 12$ & $295 \pm 15$ & $499 \pm 25$ & $1380 \pm 140$ \\
\hline $120715.46+595342.9$ & $167.0 \pm 8.3$ & $146.0 \pm 7.3$ & $174.0 \pm 8.7$ & $324 \pm 16$ & $1500 \pm 75$ \\
\hline $121221.56+534128.0$ & $239 \pm 12$ & $281 \pm 14$ & $405 \pm 20$ & $835 \pm 42$ & $2650 \pm 130$ \\
\hline $123743.08+630144.9$ & $132.0 \pm 6.6$ & $139 \pm 7$ & $197.0 \pm 9.9$ & $405 \pm 20$ & $1450 \pm 73$ \\
\hline $130216.13+003032.1^{\mathrm{a}}$ & $75.2 \pm 3.8$ & $58.1 \pm 2.9$ & $60.2 \pm 3.0$ & $79.1 \pm 4.0$ & $420 \pm 21$ \\
\hline $130332.42+621900.3$ & $89.2 \pm 4.5$ & $72.2 \pm 3.6$ & $95.1 \pm 4.8$ & $151.0 \pm 7.6$ & $552 \pm 28$ \\
\hline $133219.66+622716.0$ & $103.0 \pm 5.1$ & $102.0 \pm 5.1$ & $127.0 \pm 6.4$ & $212 \pm 11$ & $753 \pm 38$ \\
\hline $133422.63+475033.6$ & $120 \pm 6$ & $97.7 \pm 4.9$ & $107.0 \pm 5.3$ & $152.0 \pm 7.6$ & $901 \pm 45$ \\
\hline $133550.81+353315.8$ & $67.1 \pm 3.4$ & $67.8 \pm 3.4$ & $50.5 \pm 2.5$ & $54.8 \pm 2.8$ & $417 \pm 21$ \\
\hline $135249.82-031354.3$ & $165.0 \pm 8.2$ & $151.0 \pm 7.5$ & $170.0 \pm 8.5$ & $261 \pm 13$ & $1370 \pm 68$ \\
\hline $140300.22+432805.4$ & $90.7 \pm 4.5$ & $67.8 \pm 3.4$ & $82.3 \pm 4.1$ & $136.0 \pm 6.8$ & $796 \pm 40$ \\
\hline $140850.92+020522.7^{\mathrm{a}}$ & $87.1 \pm 4.4$ & $73.1 \pm 3.7$ & $73.0 \pm 3.7$ & $141.0 \pm 7.1$ & $530 \pm 27$ \\
\hline $144231.72+011055.3^{\mathrm{a}}$ & $37.8 \pm 1.9$ & $39.8 \pm 2.0$ & $34.1 \pm 1.7$ & $69.4 \pm 3.5$ & $325 \pm 16$ \\
\hline $153259.96-003944.1^{\mathrm{a}, \mathrm{b}}$ & $81.0 \pm 4.1$ & $72.6 \pm 3.6$ & $83.2 \pm 6.1$ & $123.0 \pm 6.1$ & $502 \pm 25$ \\
\hline $154734.95+444652.5$ & $84.6 \pm 4.2$ & $71.3 \pm 3.6$ & $72.3 \pm 3.6$ & $106.0 \pm 5.3$ & $406 \pm 20$ \\
\hline
\end{tabular}

Notes.

a Spitzer photometry from DS09.

b Flux densities from PSF photometry; see Section 2.2 for details.

Table 4

New Near-IR Photometry from the ARC 3.5 m Telescope

\begin{tabular}{lccc}
\hline \hline $\begin{array}{l}\text { Quasar } \\
\text { (SDSS J) }\end{array}$ & $\begin{array}{c}J \\
(\mathrm{mag})\end{array}$ & $\begin{array}{c}H \\
(\mathrm{mag})\end{array}$ & $\begin{array}{c}K_{\mathrm{S}} \\
(\mathrm{mag})\end{array}$ \\
\hline $121221.56+534128.0$ & $17.23 \pm 0.02$ & $16.80 \pm 0.03$ & $16.13 \pm 0.08$ \\
$123743.08+630144.9$ & $17.61 \pm 0.02$ & $17.18 \pm 0.04$ & $16.53 \pm 0.09$
\end{tabular}

our WLQs; the fluxes at the longest wavelengths are significantly above the power-law SED in each source. To account for this deviation, we added a single blackbody component to the powerlaw model; the best-fit models are shown on top of the WLQ SEDs in Figure 2 and Table 6 presents the best-fit parameters. With the exception of SDSS J1207+5953, the best-fit powerlaw indices are in the range $0.42 \leqslant \alpha_{\lambda} \leqslant 1.21$ and the best-fit blackbody temperatures are in the range $820 \leqslant T \leqslant 1050 \mathrm{~K}$. Due to the limited number of photometric data points available for the fits, the best-fit parameters have an uncertainty of $\sim 15 \%$. Figure 3 shows a composite SED of optically luminous quasars from R06 plotted on top of the SED of each WLQ in our sample; the R06 composite SED was vertically shifted to provide the best-fit model to each WLQ SED. It is apparent that every WLQ SED is, qualitatively, consistent with the SED of an ordinary quasar. SDSS J1207+5953 with $\alpha_{\lambda}=0.04$ may suffer from intrinsic reddening and the weakness of its emission lines may be at least partly attributed to this effect (see also Figures 2 and 3). Furthermore, this source has recently been found to show a hint of a broad C IV absorption trough and was therefore classified as a BAL quasar ${ }^{18}$ (Trump et al. 2006; Gibson et al. 2009; see Figure 1); this is the only known BAL quasar in our sample. We have therefore excluded this source from our composite WLQ SED (Section 3.2).

We note that the rest-frame UV spectra, near-IR photometry, and Spitzer photometry were obtained at different epochs,

\footnotetext{
18 The possible BAL quasar nature of this source was not known during the
} Spitzer target selection process. spaced by $\approx 1$ yr in the rest frame. These temporal gaps, however, should not affect the shapes of individual SEDs significantly assuming that WLQs vary as luminous, high-redshift quasars (which do not vary by more than $\sim 10 \%$ on such timescales; e.g., Kaspi et al. 2007; see also Section 1 and DS09) and considering that the uncertainties on our fluxes are on the order of $\sim 5 \%$.

\subsection{Composite WLQ SED}

We create a composite WLQ UV-to-mid-IR SED from the individual SEDs of all our sources, excluding SDSS J1207+5953 (see Section 3.1). The data from the 17 WLQs were binned into six rest-frame UV, two near-IR (i.e., the ground-based observations), and seven near-to-mid-IR (i.e., Spitzer) flux bins. The seven Spitzer bins include five covering the four IRAC bands, with a minimum of 10 sources per bin, and two bins covering the MIPS $24 \mu \mathrm{m}$ band with a minimum of 8 sources per bin. The flux of the composite SED in each bin represents the flux-weighted mean of all the individual SEDs contributing to that bin using the average flux densities in the $40 \AA$ wide bin centered on rest-frame $1445 \AA$ in each source. The fluxes of the composite SED were normalized by the mean flux density at rest-frame $1445 \AA$. The central wavelength of each Spitzer bin is the flux-density-weighted mean rest-frame wavelength of the data points contributing to that bin. The composite WLQ SED data are provided in Table 7 and shown in Figure 4.

Figure 4 shows that the composite WLQ SED is well represented by a model consisting of a single power law and a single blackbody; a least-squares fit results in a power-law index of $\alpha_{\lambda}=1.09 \pm 0.16$ and a blackbody temperature of $T=960 \pm 140 \mathrm{~K}$; the separate best-fit model components, i.e., a power law and a blackbody, are also shown. Also shown in Figure 4 are the R06 composite SED of optically luminous quasars and a parabolic model representing a typical LBL SED from Nieppola et al. (2006); it is clear that the WLQ SED cannot be represented by an LBL SED. The R06 quasar SED provides a good representation of the composite WLQ SED at 

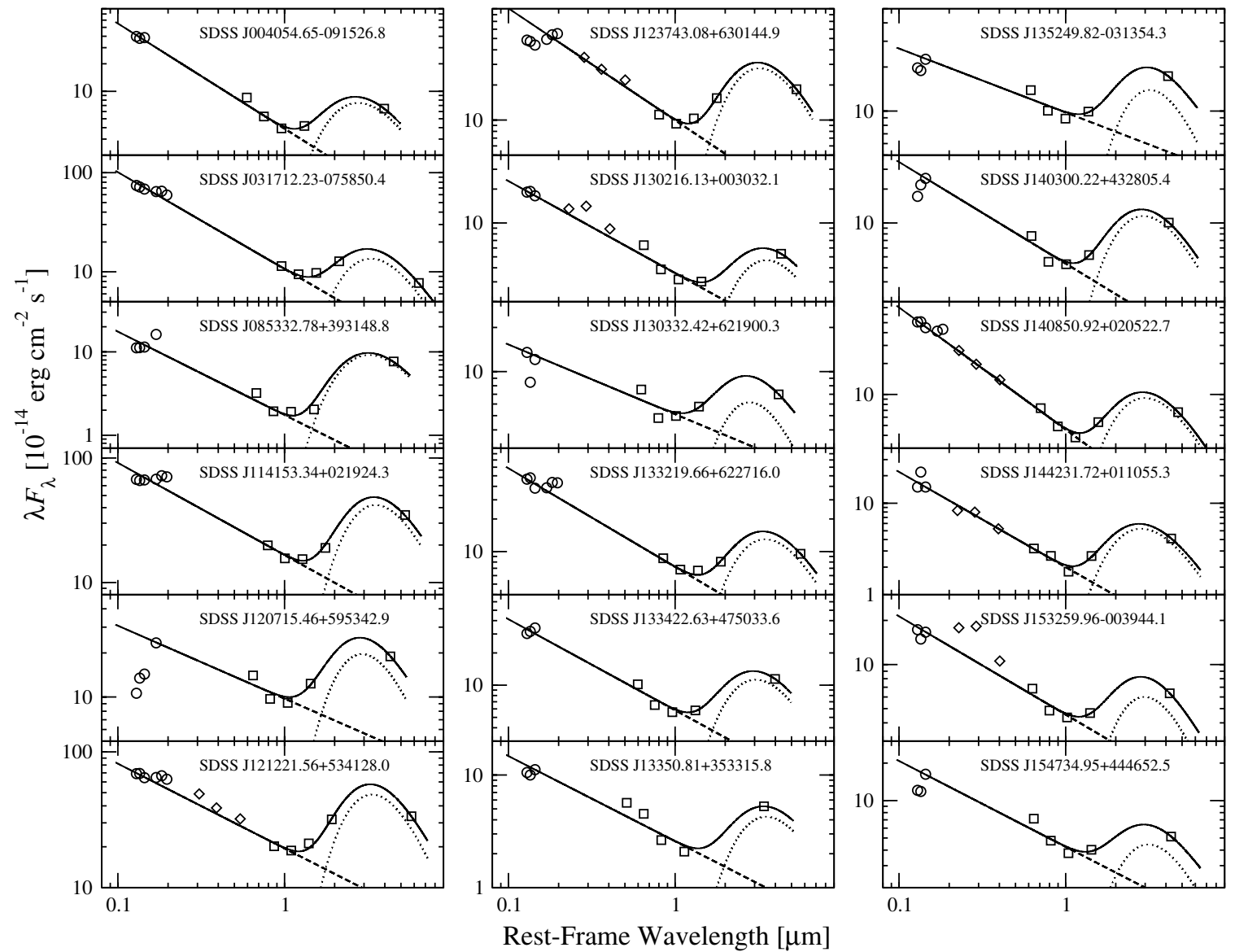

Figure 2. Rest-frame UV-to-mid-IR SEDs of our WLQs. The rest-frame UV bins are marked with circles, the observed-frame near-IR photometry, where available, are marked with diamonds, and the Spitzer photometric data are marked with squares; symbol sizes are larger than the uncertainties on the data. A best-fit power-law model (dashed line), a blackbody model (dotted line), and a power law plus blackbody model (solid line) for each SED is shown in each panel; the best-fit parameters are listed in Table 6. Note the reddened SED of the BAL quasar SDSS J120715.46+595342.9.

Table 5

Rest-frame UV Spectroscopy for the Entire WLQ Sample

\begin{tabular}{|c|c|c|c|c|c|c|c|}
\hline \multirow{2}{*}{$\begin{array}{l}\text { Quasar } \\
\text { (SDSS J) }\end{array}$} & \multicolumn{6}{|c|}{ Flux Density $(\mu \mathrm{Jy})$} & \multirow[b]{2}{*}{$\alpha_{\nu}{ }^{\mathrm{a}}$} \\
\hline & $1290 \AA$ & $1350 \AA$ & $1445 \AA$ & $1695 \AA$ & $1830 \AA$ & $1965 \AA$ & \\
\hline $004054.65-091526.8$ & $102.3 \pm 6.1$ & $102.3 \pm 9.3$ & $111.0 \pm 17.3$ & & & & 0.76 \\
\hline $085332.78+393148.8$ & $25.2 \pm 10.3$ & $26.5 \pm 7.0$ & $28.9 \pm 12.9$ & $47.9 \pm 23.4$ & $\ldots$ & $\ldots$ & 1.25 \\
\hline $114153.34+021924.3$ & $129.9 \pm 9.9$ & $132.6 \pm 19.8$ & $143.9 \pm 7.7$ & $171.4 \pm 6.8$ & $196.6 \pm 12.5$ & $206.6 \pm 15.2$ & 1.16 \\
\hline $120715.46+595342.9$ & $25.1 \pm 3.3$ & $33.2 \pm 10.7$ & $37.9 \pm 15.6$ & $73.4 \pm 3.9$ & $\ldots$ & $\ldots$ & 1.96 \\
\hline $121221.56+534128.0$ & $121.6 \pm 5.4$ & $127.9 \pm 12.0$ & $127.5 \pm 11.3$ & $150.1 \pm 11.6$ & $166.9 \pm 8.4$ & $168.9 \pm 18.0$ & 0.83 \\
\hline $130332.42+621900.3$ & $32.9 \pm 8.0$ & $21.6 \pm 8.4$ & $33.0 \pm 11.6$ & $\ldots$ & $\ldots$ & $\ldots$ & 0.51 \\
\hline $133219.66+622716.0$ & $83.7 \pm 6.5$ & $90.2 \pm 28.6$ & $77.5 \pm 9.6$ & $91.6 \pm 4.6$ & $110.9 \pm 8.3$ & $118.3 \pm 11.7$ & 0.81 \\
\hline $133422.63+475033.6$ & $77.5 \pm 7.9$ & $85.2 \pm 14.2$ & $97.9 \pm 20.9$ & $\ldots$ & $\ldots$ & $\ldots$ & 2.07 \\
\hline $133550.81+353315.8^{\mathrm{b}}$ & $31.5 \pm 4.3$ & $31.1 \pm 7.0$ & $37.2 \pm 15.5$ & $\ldots$ & $\ldots$ & $\ldots$ & 1.48 \\
\hline $135249.82-031354.3$ & $48.8 \pm 7.1$ & $49.1 \pm 19.7$ & $62.5 \pm 9.9$ & $\ldots$ & $\ldots$ & $\ldots$ & 2.19 \\
\hline $140300.22+432805.4$ & $42.2 \pm 14.1$ & $56.0 \pm 20.9$ & $68.3 \pm 31.1$ & $\ldots$ & $\ldots$ & $\ldots$ & 2.90 \\
\hline
\end{tabular}

Notes. Unless otherwise noted, the flux densities were obtained from SDSS spectra that were averaged over $40 \AA$ wide bins centered on the given wavelength; the uncertainties represent the standard deviation within the bin.

${ }^{a}$ Power-law index from the best-fit function $f_{v} \propto v^{-\alpha_{v}}$ fitted to the flux densities.

${ }^{\mathrm{b}}$ Spectrum obtained from the Multiple Mirror Telescope.

${ }^{c}$ Spectrum obtained from the W. M. Keck Observatory. 

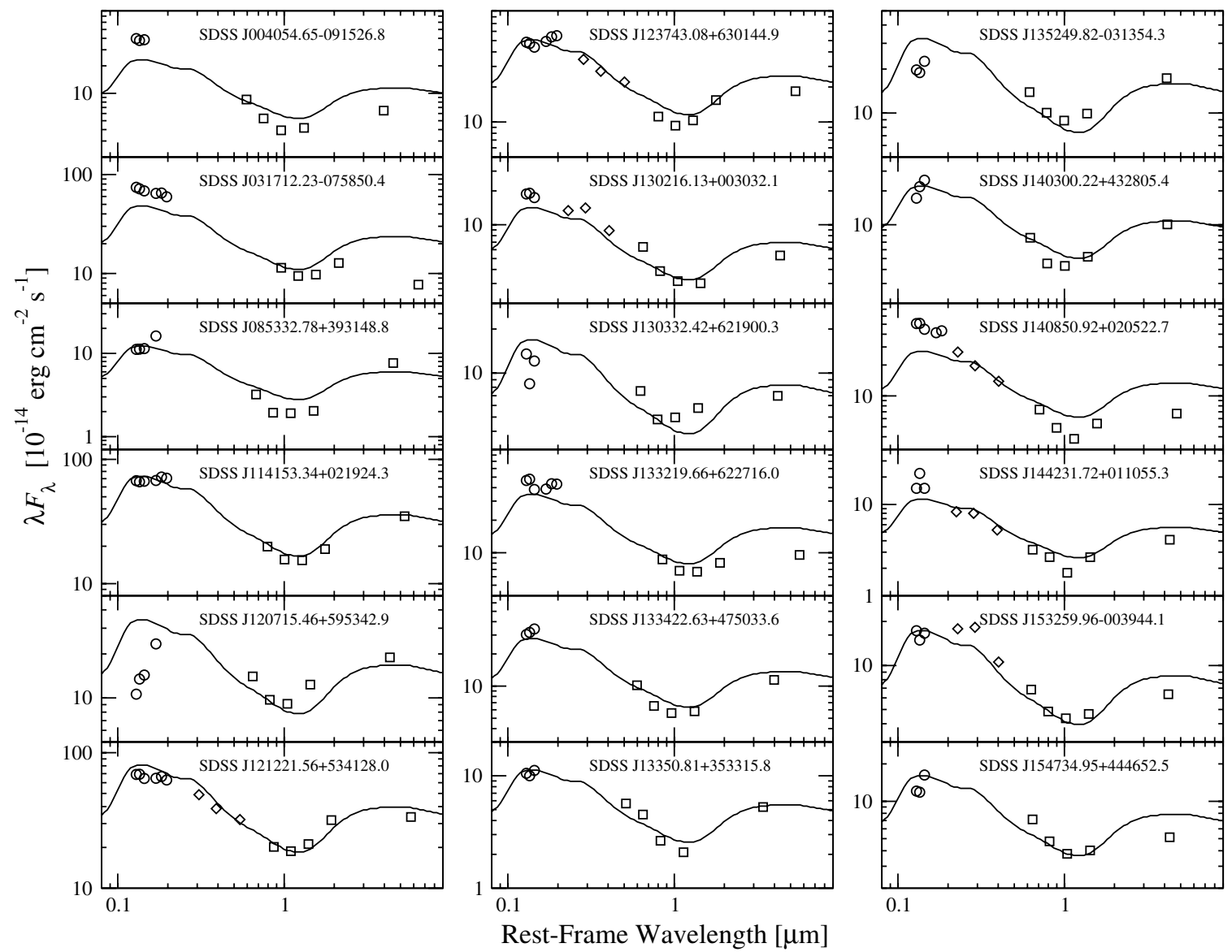

Figure 3. Rest-frame UV-to-mid-IR SEDs of our WLQs. The linearly scaled composite SED of optically luminous quasars from R06 (solid line) is shown for comparison in each panel (symbols are the same as in Figure 2). Note the discrepancy between the R06 composite SED and the SED of the BAL quasar SDSS J120715.46+595342.9 in the rest-frame UV band.

Table 6

SED Best-fit Parameters

\begin{tabular}{lcrrr}
\hline \hline $\begin{array}{l}\text { Quasar } \\
\text { (SDSS J) }\end{array}$ & $\alpha_{\lambda}{ }^{\mathrm{a}}$ & $A_{\mathrm{PL}}{ }^{\mathrm{b}}$ & \multicolumn{1}{c}{$T^{\mathrm{c}}$} & $A_{\mathrm{BB}}{ }^{\mathrm{d}}$ \\
\hline $004054.65-091526.8$ & 1.17 & 4.05 & 1050 & 7.50 \\
$031712.23-075850.4$ & 0.96 & 11.80 & 880 & 12.51 \\
$085332.78+393148.8$ & 0.89 & 1.92 & 900 & 8.93 \\
$114153.34+021924.3$ & 0.77 & 17.69 & 820 & 41.21 \\
$120715.46+595342.9$ & 0.04 & 11.21 & 990 & 11.09 \\
$121221.56+534128.0$ & 0.66 & 20.51 & 870 & 48.01 \\
$123743.08+630144.9$ & 0.89 & 10.52 & 910 & 26.82 \\
$130216.13+003032.1$ & 0.80 & 3.91 & 820 & 4.47 \\
$130332.42+621900.3$ & 0.55 & 4.50 & 1040 & 7.18 \\
$133219.66+622716.0$ & 0.94 & 7.72 & 830 & 12.74 \\
$133422.63+475033.6$ & 0.93 & 5.70 & 950 & 11.52 \\
$133550.81+353315.8$ & 0.70 & 2.95 & 820 & 4.03 \\
$135249.82-031354.3$ & 0.42 & 9.93 & 910 & 13.67 \\
$140300.22+432805.4$ & 0.85 & 4.72 & 990 & 11.15 \\
$140850.92+020522.7$ & 1.21 & 4.48 & 970 & 9.36 \\
$144231.72+011055.3$ & 1.03 & 2.03 & 1020 & 5.21 \\
$153259.96-003944.1$ & 0.81 & 4.57 & 980 & 6.49 \\
$154734.95+444652.5$ & 0.67 & 4.51 & 930 & 4.22 \\
\hline
\end{tabular}

Notes. The uncertainties on all the best-fit parameters are $\sim 15 \%$.

${ }^{a}$ Power-law index from least-squares fitting of $\lambda f_{\lambda} \propto \lambda^{-\alpha \lambda}$.

${ }^{\mathrm{b}}$ Power-law flux at rest-frame $1 \mu \mathrm{m}$ in units of $10^{-14} \mathrm{erg} \mathrm{cm}^{-2} \mathrm{~s}^{-1}$.

${ }^{c}$ Blackbody temperature.

${ }^{\mathrm{d}}$ Blackbody peak flux in units of $10^{-14} \mathrm{erg} \mathrm{cm}^{-2} \mathrm{~s}^{-1}$.
Table 7

Composite WLQ SED

\begin{tabular}{lc}
\hline \hline $\begin{array}{l}\text { Central Wavelength } \\
(\mu \mathrm{m})\end{array}$ & $\begin{array}{c}\lambda f_{\lambda} \\
\text { (Arbitrary Units) }\end{array}$ \\
\hline $0.1290_{-0.002}^{+0.002}$ & $1.030 \pm 0.087$ \\
$0.1350_{-0.002}^{+0.002}$ & $1.030 \pm 0.085$ \\
$0.1445_{-0.002}^{+0.002}$ & $1.000 \pm 0.087$ \\
$0.1695_{-0.002}^{+0.002}$ & $0.947 \pm 0.097$ \\
$0.1830_{-0.002}^{+0.002}$ & $1.030 \pm 0.112$ \\
$0.1965_{-0.002}^{+0.002}$ & $0.967 \pm 0.129$ \\
$0.2796_{-0.055}^{+0.028}$ & $0.660 \pm 0.052$ \\
$0.4450_{-0.097}^{+0.097}$ & $0.489 \pm 0.032$ \\
$0.6400_{-0.069}^{+0.069}$ & $0.319 \pm 0.039$ \\
$0.8200_{-0.078}^{+0.078}$ & $0.250 \pm 0.026$ \\
$0.9930_{-0.035}^{+0.055}$ & $0.203 \pm 0.024$ \\
$1.2300_{-0.152}^{+0.152}$ & $0.199 \pm 0.026$ \\
$1.7100_{-0.314}^{+0.413}$ & $0.244 \pm 0.036$ \\
$4.0600_{-0.641}^{+0.235}$ & $0.409 \pm 0.059$ \\
$5.5200_{-1.220}^{+0.878}$ & $0.335 \pm 0.076$ \\
\hline
\end{tabular}

Notes. Errors on the central wavelength represent the bin widths; see the text for details. Errors on $\lambda f_{\lambda}$ are the standard deviation of the flux densities in each bin. 


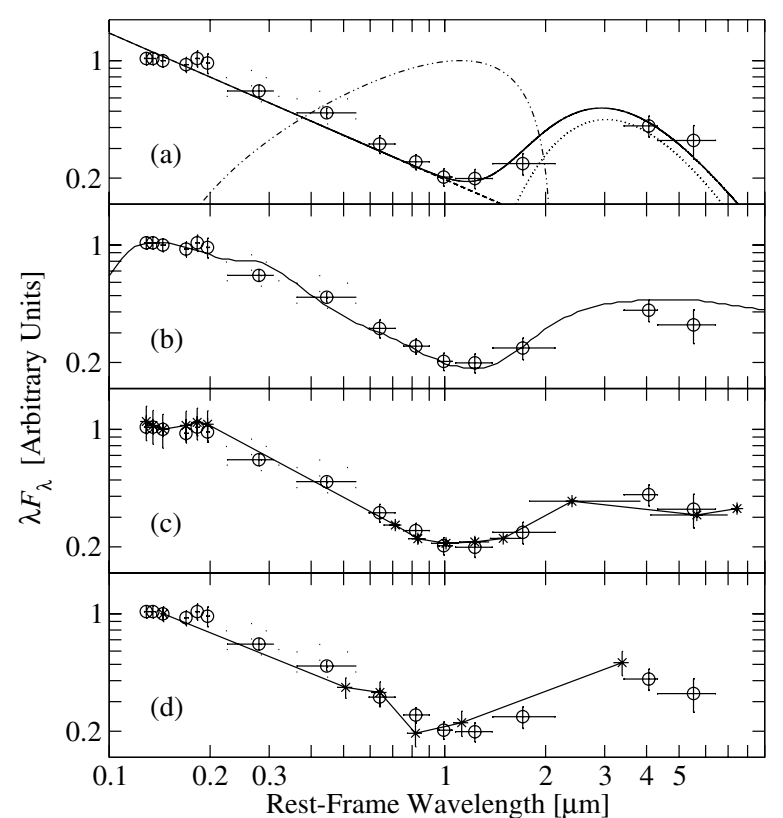

Figure 4. Our composite WLQ SED (circles) is shown against (a) the bestfit model (solid line) composed of a power-law component (dashed line) and a single blackbody component (dotted line) as well as an LBL SED model (shown for comparison; dot-dashed line), (b) the composite SED of optically luminous quasars from R06 (solid line), (c) a composite SED assembled from a redshift and luminosity matched subset of R06 quasars (asterisks connected by a solid line), and (d) a composite SED assembled from the $z \sim 6$ quasar sample of Jiang et al. (2006; asterisks connected by a solid line). In each panel, the fluxes of all SEDs are normalized to the flux at $1445 \AA$.

all wavelengths, deviating in flux by only $\sim 10 \%$ in the $J$ and MIPS $24 \mu \mathrm{m}$ bands.

To obtain a comparison between the SEDs of WLQs and ordinary quasars, we created a composite quasar SED using a subset of 22 R06 quasars that closely resemble the luminosity and redshift distributions of our WLQ sample. The redshift and luminosity ranges of this subset are $2.13 \leqslant z \leqslant 5.22$ and $45.76 \leqslant \log v L_{v}(1445 \AA) \leqslant 47.64$, respectively. The basic properties of the R06 quasar subset are given in Table 8. The composite SED for this subset was created following the method described above for the composite WLQ SED. The R06 quasar subset composite SED is shown with the composite WLQ SED in Figure 4(c). Another comparison composite UV-to-mid-IR SED was created (as described above for the composite WLQ SED) using 12 of the $13 z \sim 6$ quasars from Jiang et al. (2006). SDSS J000552.34-000655.8 was excluded from that sample because it does not have a MIPS $24 \mu \mathrm{m}$ detection (see also Jiang et al. 2010). The Jiang et al. (2006) composite SED is shown in Figure 4(d). The composite SEDs of both the R06 quasar subset and the Jiang et al. (2006) sample are consistent, within the errors (i.e., within $\sim 5 \%$ ), with our composite WLQ SED.

\section{DISCUSSION}

Our Spitzer observations have increased the number of WLQ UV-to-mid-IR SEDs by more than a factor of four, allowing us to constrain their emission mechanism in this spectral range in a statistically meaningful manner. By examining the WLQ SED in this critical spectral region we can determine if it is consistent with a relativistically boosted continuum, e.g., that of a BL Lac object, or whether it shows signatures of a heated dust mid-IR "bump" characteristic of ordinary quasars. Our
Table 8

Richards et al. (2006) Quasar Subset

\begin{tabular}{lcc}
\hline \hline $\begin{array}{l}\text { Quasar } \\
\text { (SDSS J) }\end{array}$ & $z$ & $\begin{array}{c}\log v L_{v}(1445 \AA) \\
\left(\mathrm{erg} \mathrm{s}^{-1}\right)\end{array}$ \\
\hline $103147.64+575858.0$ & 2.244 & 46.34 \\
$103628.15+585832.1$ & 3.249 & 46.41 \\
$103750.59+590132.1$ & 2.126 & 46.20 \\
$103800.50+582343.0$ & 3.933 & 46.48 \\
$103952.54+573303.2$ & 2.370 & 46.70 \\
$104018.52+572448.1$ & 5.215 & 47.30 \\
$104514.61+593707.3$ & 4.400 & 46.84 \\
$104639.42+584047.8$ & 2.961 & 45.96 \\
$104809.19+570242.0$ & 5.132 & 47.64 \\
$105036.47+580424.6$ & 2.697 & 46.09 \\
$105322.98+580412.1$ & 3.180 & 46.72 \\
$105524.56+580957.3$ & 3.064 & 46.24 \\
$105654.96+583712.4$ & 2.417 & 46.32 \\
$105715.49+573324.3$ & 3.854 & 46.93 \\
$105902.04+580848.7$ & 2.139 & 45.85 \\
$110041.96+580001.0$ & 3.409 & 45.76 \\
$161238.27+532255.0$ & 2.243 & 46.34 \\
$164022.78+411548.1$ & 2.879 & 46.33 \\
$164105.35+403651.7$ & 2.471 & 45.76 \\
$164238.08+412104.7$ & 4.771 & 47.12 \\
$171652.34+590200.1$ & 3.123 & 46.54 \\
$172358.01+601140.0$ & 2.445 & 46.53 \\
\hline & &
\end{tabular}

WLQs have UV-to-mid-IR SEDs that are well fitted by a model consisting of a power law plus blackbody with a power-law index of $\alpha_{\lambda} \sim 1$ and a blackbody temperature of $T \sim 1000 \mathrm{~K}$; these parameters are typical of the emissions from a quasar accretion disk and circumnuclear heated dust, respectively (e.g., Elvis et al. 1994; R06). None of the SEDs is consistent with pure synchrotron emission either from an HBL or an LBL, as all SEDs show a significant departure from a pure power-law model at $\lambda_{\text {rest }} \gtrsim 1 \mu \mathrm{m}$ and none can be fitted with a parabolic function. These results set an upper limit of $\approx 5 \%$ on the potential BL Lac "contamination" fraction in WLQ samples.

The R06 optically luminous quasar composite SED provides an adequate fit to the composite WLQ SED, and SED subsets composed from ordinary quasars with luminosities and redshifts similar to our WLQs (from R06 and Jiang et al. 2006) are even better, matching our composite WLQ SED within the uncertainties (Figure 4). This match provides conclusive evidence against the possibility that the emission lines in WLQs are overwhelmed by a relativistically boosted continuum. The emission lines in WLQs must therefore be intrinsically weak.

The evidence from their UV-to-mid-IR SED, combined with their X-ray and radio properties (DS09; S09) as well as their variability and polarization properties (DS09), demonstrates, unambiguously, that WLQs are unbeamed quasars with extreme emission line properties. The composite WLQ SED indicates that, at least in the rest-frame $\sim 0.1-5 \mu \mathrm{m}$ spectral range, the broadband properties of WLQs are indistinguishable from those of quasars with similar luminosities and redshifts, in spite of the significant difference in emission line strength. While the reason for the discrepancy between the broadband spectral properties and the emission line properties in WLQs is still not understood, our results clearly show that WLQs cannot be selected based on their broadband continuum properties alone, for example, by traditional photometric surveys in any band. The selection of WLQs is currently feasible only with spectroscopic surveys such as the SDSS (e.g., Plotkin et al. 2010a). 
Our results suggest that the two most probable explanations for the weakness of the UV emission lines of WLQs are either a deficiency of energetic ionizing photons, or a deficiency of line-emitting gas in the BELR. The first could be a result of a UV-peaked SED (i.e., intrinsic X-ray weakness) that may be a consequence of an extremely high accretion rate. For example, this may be the case with the high accretion rate quasar PHL 1811 at $z=0.19$ which is X-ray weaker by a factor of ¿50 than ordinary quasars with matched optical luminosity (Leighly et al. 2007b; Gibson et al. 2008). No WLQ so far observed with sensitive X-ray imaging is X-ray weaker than PHL 1811 (although a few WLQs have sensitive X-ray upper limits and could potentially be weaker; S09; Wu et al. 2011). The peculiar, soft SED of PHL 1811 can naturally explain why the high-ionization emission lines (such as C IV) of the source are unusually weak relative to their low-ionization counterparts (e.g., $\mathrm{H} \beta$; Leighly et al. 2007a, 2007b).

However, the extremely high accretion rate scenario for WLQs faces several difficulties. An extremely high accretion rate would have been expected to result in suppressed IR luminosity with respect to the bolometric luminosity of the accretion disk (e.g., Maiolino et al. 2007; Kawakatu \& Ohsuga 2011). We do not have any evidence for significant suppression of IR continuum emission in our WLQs. In fact, the SEDs of WLQs and ordinary quasars are nearly indistinguishable in the $\sim 0.1-5 \mu \mathrm{m}$ rest-frame band across broad luminosity and redshift ranges for the ordinary quasars; any remaining differences disappear when more comparable luminosity and redshift ranges are considered. Moreover, the X-ray properties of at least a subset of our WLQs are similar to the X-ray properties of ordinary quasars with comparable luminosities (S09). It is therefore reasonable to assume that the distributions of the normalized accretion rates (in terms of the Eddington luminosity ratio, $L / L_{\mathrm{Edd}}$, where $L$ and $L_{\mathrm{Edd}}$ represent the bolometric and Eddington luminosities, respectively) of WLQs and ordinary quasars are similar as well. Recently, Shemmer et al. (2010) have directly determined the $L / L_{\text {Edd }}$ values for two WLQs from our sample, SDSS J1141+0219 and SDSS J1237+6301, and found these to be typical of ordinary quasars. In addition, these WLQs exhibit exceptionally weak $\mathrm{H} \beta$ lines, comparable to the extreme weakness of their UV lines. This result favors the scenario in which the weakness of the WLQ emission lines, across the spectrum, may be attributed to a BELR with abnormal properties, such as a low BELR covering factor, but clearly no firm conclusions can be drawn based on only two sources.

Another possibility for obtaining a deficiency of high-energy photons, albeit with a typical underlying quasar SED, is based on a recent investigation of 10 SDSS quasars at $z \simeq 2.2$ selected to have UV line properties that are similar to those of PHL 1811 (hereafter "PHL 1811 analogs"; Wu et al. 2011). Wu et al. (2011) suggest that the weakness of the UV lines in their PHL 1811 analogs is due to a larger than usual high-ionization "shielding gas" component that covers most of the BELR, but little more than the BELR in such sources. In this scenario, such PHL 1811 analogs are a subset of WLQs, viewed through the shielding gas while "normal" WLQs are viewed at lines of sight that do not intersect this component. This may explain the observed X-ray weakness and apparently hard X-ray spectra of the PHL 1811 analogs (Wu et al. 2011), although it is more difficult to explain the very steep (soft) X-ray spectrum of PHL 1811 itself using this model (Leighly et al. 2007b).

Although the evidence from the radio through X-ray broadband continua of WLQs as well as the few observations of their rest-frame optical spectra favor the "abnormal BELR" scenario, one cannot draw definitive conclusions about the nature of WLQs without additional observations. Near-IR spectroscopy of a statistically meaningful sample of WLQs will determine both whether their low-ionization emission lines are as weak as their high-ionization counterparts and whether their accretion rates are exceptionally high. Additional X-ray spectroscopy of these WLQs can then test the reliability of such $L / L_{\text {Edd }}$ determinations if their BELRs are indeed abnormal (see, e.g., Shemmer et al. 2010). Extended and more detailed coverage of the WLQ SED is also required to allow comparisons between predicted and observed emission line EWs. For example, the current $\sim 0.1-5 \mu \mathrm{m}$ WLQ SED is only sparsely covered; considerably denser sampling, in particular in the near-IR band, would bridge the gap between the available SDSS spectroscopy and Spitzer photometry. Such observations, coupled with photoionization modeling, would improve our understanding of the conditions necessary for emission line formation in all AGNs.

\section{SUMMARY}

We present new Spitzer observations of 14 high-redshift $(z>2.2)$ quasars with weak or undetectable emission lines in their rest-frame UV spectra (WLQs) as well as new groundbased, near-IR photometry of two of these sources. Together with archival Spitzer and near-IR photometry as well as restframe UV spectroscopy, these observations allowed us to trace the UV-mid-IR SEDs (i.e., $\sim 0.1-5 \mu \mathrm{m}$ ) of a statistically representative sample of 18 of the $\sim 80$ known WLQs. All the SEDs show a clear indication of a typical quasar near-to-mid-IR heated dust "bump" and they are well represented by a model consisting of a power law plus blackbody; the ranges of best-fit power-law indices $\left(0.04 \leqslant \alpha_{\lambda} \leqslant 1.21\right)$ and blackbody temperatures $(820 \leqslant T \leqslant 1050)$ are comparable to the ranges observed in SEDs of ordinary quasars. We find that all individual SEDs are inconsistent with a relativistically boosted continuum that might have been expected if WLQs would have been associated with BL Lac objects. By comparing a composite WLQ SED of 17 sources (i.e., excluding SDSS J1207+5953 that has a hint of a C IV BAL trough) with composite SEDs of ordinary quasars with matched luminosities and redshifts, we conclude that WLQs are unbeamed quasars and that their UV emission lines are intrinsically weak. The similarity in broadband continuum properties between WLQs and ordinary quasars, in contrast with the significant differences between their UV emission line strengths, suggests that WLQs can only be selected efficiently in spectroscopic surveys. We discuss different scenarios for explaining the weakness of the UV lines in WLQs, mainly extremely high accretion rates or abnormal BELRs, and describe additional observations required to test both hypotheses.

This work is based on observations made with the Spitzer Space Telescope, which is operated by the Jet Propulsion Laboratory, California Institute of Technology, under a contract with NASA. Support for this work was provided by NASA through an award issued by JPL/Caltech. The work is based in part on observations obtained with the Apache Point Observatory $3.5 \mathrm{~m}$ telescope, which is owned and operated by the Astrophysical Research Consortium. We gratefully acknowledge financial support from the University of North Texas Research Initiation Grants G34029 and GA9560 (R.A.L., O.S.), from the Southern California Center for Galaxy Evolution, a multi-campus research program funded by the University of California Office of Research (A.M.D.), from a NASA ADP 
grant NNX10AC99G (W.N.B.), and from an NSF grant AST0707266 (M.A.S.). We thank an anonymous referee for a helpful report, and the members of the Spitzer helpdesk team for assistance with the data reduction. This research has made use of the NASA/IPAC Extragalactic Database (NED) which is operated by the Jet Propulsion Laboratory, California Institute of Technology, under contract with the National Aeronautics and Space Administration. Funding for the SDSS and SDSS-II has been provided by the Alfred P. Sloan Foundation, the Participating Institutions, the National Science Foundation, the U.S. Department of Energy, the National Aeronautics and Space Administration, the Japanese Monbukagakusho, the Max Planck Society, and the Higher Education Funding Council for England. The SDSS Web site is http://www.sdss.org/.

Facilities: Spitzer, Sloan, ARC

\section{REFERENCES}

Anderson, S. F., Fan, X., Richards, G. T., et al. 2001, AJ, 122, 503 Becker, R. H., White, R. L., \& Helfand, D. J. 1995, ApJ, 450, 559 Cardelli, J. A., Clayton, G. C., \& Mathis, J. S. 1989, ApJ, 345, 245 Collinge, M. J., Strauss, M. A., Hall, P. B., et al. 2005, AJ, 129, 2542 Condon, J. J., Cotton, W. D., Greisen, E. W., et al. 1998, AJ, 115, 1693

Diamond-Stanic, A. M., Fan, X., Brandt, W. N., et al. 2009, ApJ, 699, 782 (DS09)

Elvis, M., Wilkes, B. J., McDowell, J. C., et al. 1994, ApJS, 95, 1

Fan, X., Strauss, M. A., Gunn, J. E., et al. 1999, ApJ, 526, L57

Fan, X., Strauss, M. A., Richards, G. T., et al. 2001, AJ, 121, 31

Fan, X., Strauss, M. A., Richards, G. T., et al. 2006, AJ, 131, 1203

Fazio, G. G., Hora, J. L., Allen, L. E., et al. 2004, ApJS, 154, 10

Fossati, G., Maraschi, L., Celotti, A., Comastri, A., \& Ghisellini, G. 1998, MNRAS, 299, 433

Gibson, R. R., Brandt, W. N., \& Schneider, D. P. 2008, ApJ, 685, 773

Gibson, R. R., Jiang, L., Brandt, W. N., et al. 2009, ApJ, 692, 758

Hryniewicz, K., Czerny, B., Nikołajuk, M., \& Kuraszkiewicz, J. 2010, MNRAS, 404, 2028
Jannuzi, B. T., Green, R. F., \& French, H. 1993, ApJ, 404, 100

Jiang, L., Fan, X., Brandt, W. N., et al. 2010, Nature, 464, 380

Jiang, L., Fan, X., Hines, D. C., et al. 2006, AJ, 132, 2127

Kaspi, S., Brandt, W. N., Maoz, D., et al. 2007, ApJ, 659, 997

Kawakatu, N., \& Ohsuga, K. 2011, arXiv:1107.2185

Kellermann, K. I., Sramek, R., Schmidt, M., Shaffer, D. B., \& Green, R. 1989, AJ, 98, 1195

Leighly, K. M., Halpern, J. P., Jenkins, E. B., \& Casebeer, D. 2007a, ApJS, 173, 1

Leighly, K. M., Halpern, J. P., Jenkins, E. B., et al. 2007b, ApJ, 663, 103

Liu, Y., \& Zhang, S. N. 2011, ApJ, 728, L44

Londish, D., Heidt, J., Boyle, B. J., Croom, S. M., \& Kedziora-Chudczer, L. 2004, MNRAS, 352, 903

Maiolino, R., Shemmer, O., Imanishi, M., et al. 2007, A\&A, 468, 979

Makovoz, D., \& Marleau, F. R. 2005, PASP, 117, 1113

McDowell, J. C., Canizares, C., Elvis, M., et al. 1995, ApJ, 450, 585

Nieppola, E., Tornikoski, M., \& Valtaoja, E. 2006, A\&A, 445, 441

Oke, J. B., Cohen, J. G., Carr, M., et al. 1995, PASP, 107, 375

Padovani, P., \& Giommi, P. 1995, ApJ, 444, 567

Plotkin, R. M., Anderson, S. F., Brandt, W. N., et al. 2010a, AJ, 139, 390

Plotkin, R. M., Anderson, S. F., Brandt, W. N., et al. 2010b, ApJ, 721, 562

Richards, G. T., Lacy, M., Storrie-Lombardi, L. J., et al. 2006, ApJS, 166, 470 (R06)

Rieke, G. H., Young, E. T., Engelbracht, C. W., et al. 2004, ApJS, 154, 25

Schlegel, D. J., Finkbeiner, D. P., \& Davis, M. 1998, ApJ, 500, 525

Schneider, D. P., Hall, P. B., Richards, G. T., et al. 2005, AJ, 130, 367

Shemmer, O., Brandt, W. N., Anderson, S. F., et al. 2009, ApJ, 696, 580 (S09)

Shemmer, O., Brandt, W. N., Schneider, D. P., et al. 2006, ApJ, 644, 86 (S06)

Shemmer, O., Trakhtenbrot, B., Anderson, S. F., et al. 2010, ApJ, 722, L152

Skrutskie, M. F., Cutri, R. M., Stiening, R., et al. 2006, AJ, 131, 1163

Stocke, J. T., Morris, S. L., Gioia, I., et al. 1990, ApJ, 348, 141

Stocke, J. T., \& Perrenod, S. C. 1981, ApJ, 245, 375

Trump, J. R., Hall, P. B., Reichard, T. A., et al. 2006, ApJS, 165, 1

Urry, C. M., \& Padovani, P. 1995, PASP, 107, 803

Vanden Berk, D. E., Richards, G. T., Bauer, A., et al. 2001, AJ, 122, 549

Werner, M. W., Roellig, T. L., Low, F. J., et al. 2004, ApJS, 154, 1

Wu, J., Brandt, W. N., Hall, P. B., et al. 2011, ApJ, 736, 28

York, D. G., Adelman, J., Anderson, J. E., Jr., et al. 2000, AJ, 120, 1579 